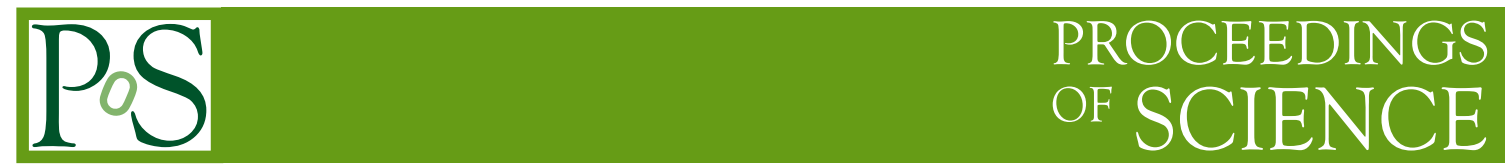

\title{
Minimum Bias and Hadronic Event Shapes at LHC
}

\author{
I.Vivarelli ${ }^{* \dagger}$ \\ Albert-Ludwigs Universität, Freiburg \\ E-mail: iacopo.vivarelliecern.ch
}

\begin{abstract}
Given the large uncertainties in the extrapolation at the LHC energies of the soft hadron physics cross sections and in the particle multiplicities, the direct measurement of the minimum bias and the underlying event at LHC will be necessary for the understanding of the Standard Model and (possibly) Beyond the Standard Model physics. We review the plans of the ATLAS and CMS to perform such measurements at the beginning of the LHC data taking.
\end{abstract}

European Physical Society Europhysics Conference on High Energy Physics, EPS-HEP 2009,

July 16 - 222009

Krakow, Poland

\footnotetext{
*Speaker.

${ }^{\dagger}$ On behalf of the ATLAS and CMS collaborations
} 

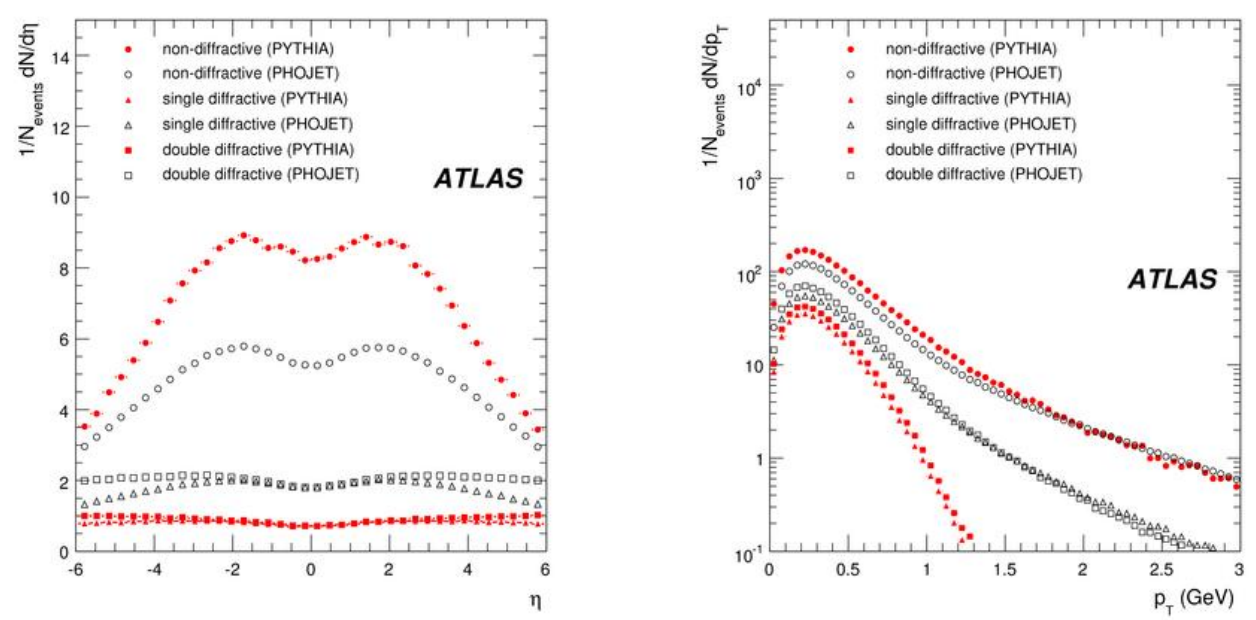

Figure 1: Number of primary tracks per unit pseudorapidity (left) and per unit $P_{T}$ (right) for single diffractive, double diffractive, non diffractive $p p$ events at $14 \mathrm{TeV}$ as predicted by the Pythia and PHOJET generators.

\section{Introduction}

The early understanding of the soft hadron interaction at the LHC energies will play a key role at the two LHC general purpose experiments, ATLAS and CMS. The uncertainties in the underlying event, arising mainly from the multiple parton-parton interaction in a $p p$ collision, and in the multiple proton-proton collisions per bunch crossing (the pile-up from minimum bias) will contribute to the uncertainties on the jet and missing $E_{T}$ scale, on the lepton isolation criteria, vertexing, etc.

The cross section of the minimum bias events is expected to be about $80 \mathrm{mb}$ (at $14 \mathrm{TeV}$ in the CM), implying up to 25 (for a luminosity of $10^{34} \mathrm{~cm}^{-2} \mathrm{~s}^{-1}$ with a bunch spacing of $25 \mathrm{~ns}$ ) minimum bias events superimposed to the high $P_{T}$ collision. The uncertainty of the extrapolation from the Tevatron energies up to those of the LHC (in terms of cross section, track multiplicity and spectra) will force ATLAS and CMS to measure the minimum bias (and the underlying event) with the first data available.

In this paper we review the strategies of the two experiments for an early determination of the soft hadron interactions.

\section{Minimum Bias Determination}

Figure 1 shows the pseudorapidity and $P_{T}$ track spectra for single diffractive, double diffractive and non diffractive events obtained with Pythia 6.401 [1] and PHOJET [2]. The tuning of both generators is such that the data from lower energy experiments are reproduced. Some considerations can be made: 

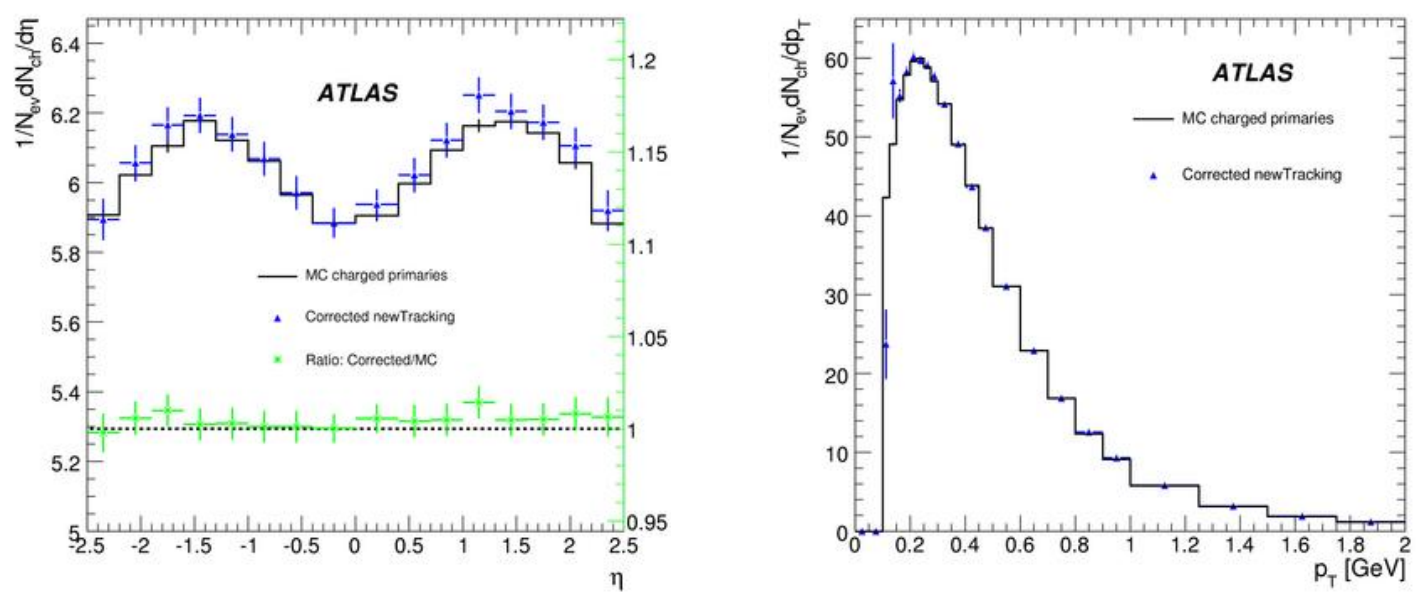

Figure 2: Comparison between the input generator events and the reconstructed pseudorapidity (left) and $P_{T}$ (right) distributions for non single diffractive events.

- The two different generators give different predicition for the track multiplicity and $P_{T}$. The two experiments cannot simply rely on the extrapolated prediction from lower energy.

- The bulk of the $P_{T}$ spectrum lies at low $P_{T}$ (the peak is at about $200 \mathrm{MeV}$ ). The detectors must be efficient in measuring low $P_{T}$ tracks.

A large effort has been devoted to minimize the detector biases for the track spectra measurement, arising mainly from the trigger and from the low $P_{T}$ tracking.

As soon as the number of collisions per bunch crossing is about one, ATLAS and CMS forsee to have an unbiased, random trigger for minimum bias events. However, especially in the early running, the number of collisions per bunch crossing is expected to be significantly lower than one. In order to reject empty events, ATLAS set up a dedicated trigger, which makes use of dedicated Minimum Bias Trigger Scintillators (MBTS), located on the cryostat in front of the foward calorimeter [3]. The threshold settings on the scintillators has been determined making use of cosmic ray data. CMS [4] is instead planning on triggering on the number of the forward calorimeter towers above a threshold.

The ATLAS measurement [5] of the minimum bias track pseudorapidity and $P_{T}$ spectra is based on track counting. The threshold for the track reconstruction has been lowered to about $150 \mathrm{MeV}$ (from the nominal $500 \mathrm{MeV}$ ) basically using only the seven silicon layers of the inner detector, thus removing the requirement of any hit in the Transition Radiation Tracker [3]. The track selection is based on the number of hits in the innermost silicon layer, on track quality and track-to-vertex association criteria. Such cuts remove most (95\%) of the secondary tracks, with the drawback of removing about $25 \%$ of the primary tracks. Additional corrections (determined making use of the detector simulation) are thus needed to take into account the track selection and vertexing efficiency.

The comparison between the generator input and the reconstructed spectra after detector simulation, reconstruction, track selection and analysis corrections (determined on an independent 

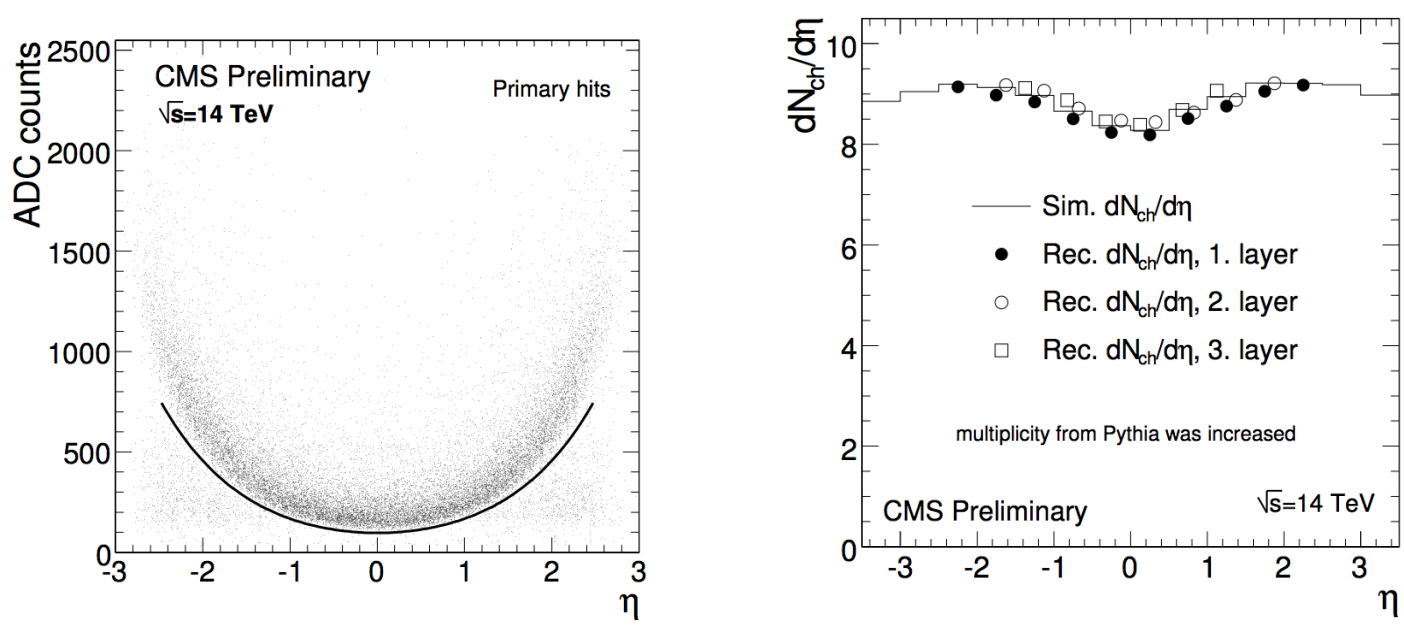

Figure 3: Left: The ADC signal in the pixel modules for hits associated to primary tracks as a function of the pseudorapidity. Right: reconstructed pseudorapidity spectrum after the hits-to-track corrections.

simulated sample) is shown in fig. 2. The reconstructed distributions agree well with those from the generator. A detailed study of the systematics shows that the dominant uncertainty is that associated with the inner detector alignment. Different alignment conditions have been used to generate different data samples. The associated systematics is quoted to be $6 \%$, while the total systematics (including track corrections uncertainties, possible vertex biases, etc.) is expected to be $8 \%$.

In order to reduce the dependency on the detector alignment, CMS has developed two complementary strategies that will be used for the first data. The first analysis is based on hits counting [6]. Hits coming from primary tracks show a correlation between their pseudorapidity position and the ionization energy deposits in the pixel module (see fig. 3, left). Such correlation can be used to separate signal hits from background hits. The event selection is done requiring the presence of at least one reconstructed vertex, with respect to which the pseudorapidity position of the hits is computed. The track multiplicity is then recovered applying a hits-to-track correction function obtained from simulated events. Figure 3 (right) shows the pseudorapidity spectrum (after the hits to track corrections) superimposed to the simulation input. A good agreement is found.

A complementary approach in CMS is based on tracklets reconstruction [7]. Tracklets are defined as pairs of hits in the first two layers of the CMS inner detector. First, proto-tracklets are reconstructed with no vertex information (using a simple $\eta-\phi$ association). Then, the primary vertex is identified using the proto-tracklets, and the final traclets are finally reconstructed. One appealing aspect of the method is that the background subtraction can be done directly from the data, looking (for example) at the $\Delta \phi=\phi_{1}-\phi_{2}$ distribution $\left(\phi_{1(2)}\right.$ is the $\phi$ of the tracklet hits on layer number 1(2), see fig. 4 (left)). The tracklet distribution is then corrected to obtain the number of primary tracks (the correction being determined on simulated data). The comparison of the results obtained after the tracklet reconstruction, the background subtraction and the final corrections is shown on Fig. 4 (right panel). The agreement between the input data and the reconstructed one is good. 

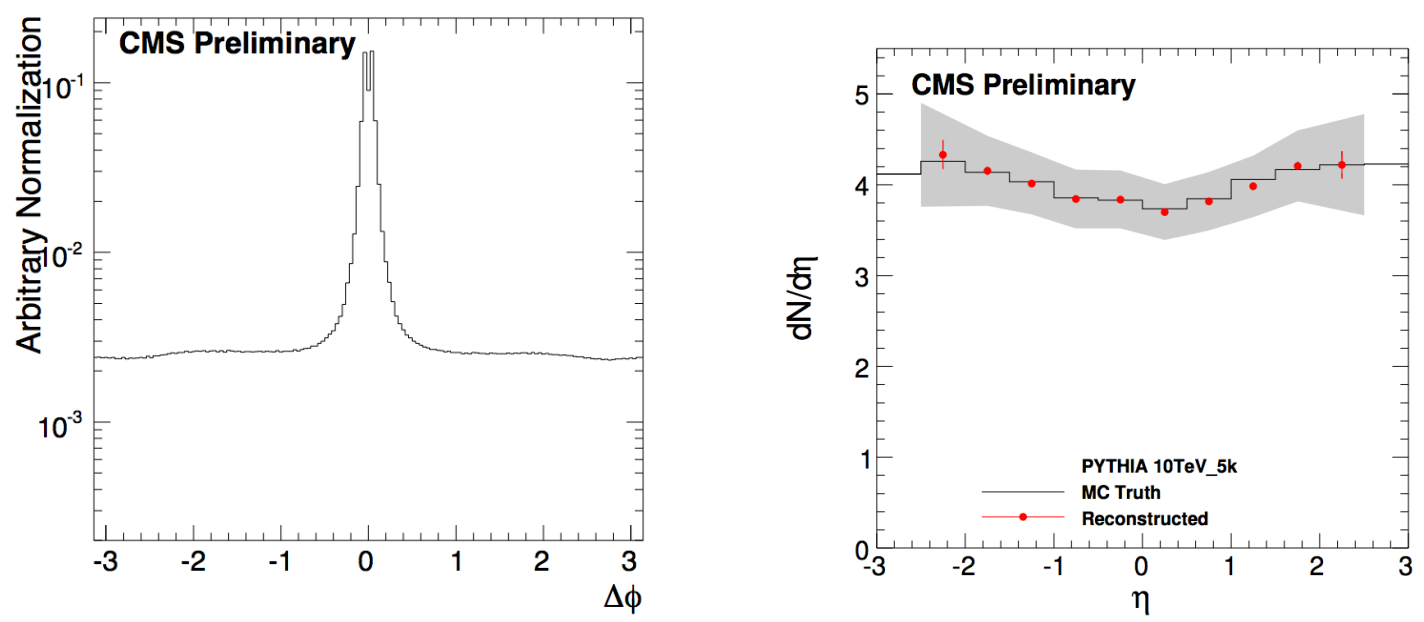

Figure 4: Left: $\Delta \phi$ distribution between the hits of the tracklets in a minimum bias sample. Right: the reconstructed data are compared with the simulation input.

\section{Conclusions}

The extrapolation of the minimum bias cross sections from the Tevatron energies to those of LHC is highly uncertain. Both the LHC experiments, ATLAS and CMS, have dedicated and well developed strategies for triggering, reconstructing and analyzing the minimum bias events. All the analysis performed so far on simulated data show that the final results obtained are robust against several effects (in particular misalignment), and that the systematics are under control.

\section{References}

[1] Sjostrand, T. and Mrenna, S. and Skands, P., JHEP 05 (2006) 026.

[2] PHOJET manual (program version 1.05c, June 96)

[3] The ATLAS Collaboration, G. Aad et al., "The ATLAS Experiment at the CERN Large Hadron Collider", JINST 3 (2008) S08003.

[4] The CMS Collaboration, S. Chatrchyan et al., "The CMS experiment at the CERN LHC", JINST 3 (2008) S08004.

[5] The ATLAS Collaboration, G.Aad et al., "Expected Performance of the ATLAS Experiment, Detector, Trigger and Physics", CERN-OPEN-2008-020.

[6] The CMS Collaboration, "Pseudorapidity distributions of charged hadrons in minimum bias $p p$ collisions a $\sqrt{s}=14 \mathrm{TeV} "$, CMS PAS QCD-08-004.

[7] The CMS Collaboration, "Study of Charged Hadron Multiplicity in Minimum Bias $p p$ Collisions at $\sqrt{s}=900 \mathrm{GeV}$ and $10 \mathrm{TeV}$ ", CMS PAS QCD-09-002. 